\title{
Responsabilidad Social Universitaria y desarrollo: una aproximación regional para Colombia
}

\author{
López Rodríguez, María del Pilar \\ Universidad de San Buenaventura Cali, Cali, Colombia \\ mplopez2@usbcali.edu.co \\ Martínez Usarralde, María Jesús \\ Universitat de València, Valencia, España \\ m.jesus.martinez@uv.es \\ Lloret Calalá, Mari Carmen \\ Universitat de València, Valencia, España \\ m.carmen.Iloret@uv.es
}

\section{Resumen}

La educación superior tiene la capacidad de contribuir a la transformación positiva de las principales situaciones-problema asociadas a la pobreza, la desigualdad, la inequidad y el deterioro del medio ambiente. En este sentido, la responsabilidad social como modelo de direccionamiento y gestión de las universidades, propende por la formación de profesionales agentes de desarrollo económico y social, cuyas metas individuales resulten compatibles con el mejoramiento de su entorno y la sostenibilidad del territorio. Esta investigación se encuentra situada en el campo disciplinar de la educación comparada, y tiene como propósito promover el acercamiento entre el discurso y la práctica de la RSU en Colombia; para ello, se parte de revisar diferentes categorías del desarrollo y sus variables identificables, a partir de las cuales se caracteriza el panorama socioeconómico y educativo de los departamentos de Colombia, y se lo relaciona con el ejercicio de la RSU en diferentes universidades del país. Dentro de los principales hallazgos se evidencia una clara correspondencia entre los perfiles regionales de desarrollo socioeconómico, los alcances educativos en el nivel superior, y la presencia de políticas de responsabilidad social en las universidades. Estas formas de relacionamiento refuerzan la hipótesis de que la RSU es un eje de articulación efectivo y pertinente en el engranaje sociedad- estado-universidad, al actuar como dinamizador de los modelos de desarrollo.

\section{Abstract}

Higher education has the capacity to contribute to the positive transformation of the main problem situations associated with poverty, inequality, and the deterioration of the environment. In this sense, social responsibility as a model of management of universities, tends to train professionals in economic and social development, whose individual goals are compatible with the improvement of their environment and the sustainability of the territory. This research is located in the disciplinary field of comparative education, and its purpose is to promote the rapprochement between the discourse and the practice of the RSU in Colombia; For this purpose, we start by reviewing different categories of development and their identifiable variables, from which the socioeconomic and educational panorama of the Colombian departments is characterized, and it is related to the exercise of the RSU in different universities of the country. Among the main findings there is a clear correspondence between the regional profiles of socioeconomic development, the educational scope at the higher level, and the presence of social responsibility policies in universities. These forms of relationship reinforce the hypothesis that the RSU is an axis of effective and pertinent articulation in the partnership society-state-university, by acting as a catalyst for development models.

Palabras clave: Responsabilidad social universitaria, desarrollo económico, desarrollo social, desarrollo humano, educación comparada.

Keywords: University social responsibility, economic development, social development, human development, comparative education. 


\section{INTRODUCCIÓN}

Sobre el desarrollo en sus diferentes acepciones (económico, sostenible, humano...) se han escrito innumerables tratados y artículos que sustentan teorías y paradigmas, en un intento por explicar los determinantes de lo que se consideraría un país "desarrollado", y por identificar el camino óptimo hacia la visión de desarrollo que se desea alcanzar; no obstante, hoy por hoy el debate sigue inconcluso, y está más vigente que nunca.

La motivación que subyace el perdurable y a la vez renovado interés en estudiar el desarrollo como fenómeno socioeconómico, se encuentra en gran medida en la necesidad que tienen las sociedades modernas de encontrar un camino certero hacia la resolución de problemáticas que se han afianzado y diversificado a lo largo del tiempo, las cuales afectan negativamente la calidad de vida de la población y deterioran su bienestar.

Al respecto, cabe preguntarse entonces ¿sobre qué o quiénes recae la responsabilidad del desarrollo de un territorio? La respuesta natural sería atribuir este deber al Estado y sus gobernantes, pero enfoques teóricos más recientes de mitad del siglo XX hasta la fecha, identifican nuevos elementos como: las instituciones (neoinstitucionalismo), la acumulación de conocimiento (capital humano) y las redes (capital social) como determinantes de la evolución socioeconómica de la población, en los que la Universidad tiene una profunda orientación transformadora.

Esta investigación parte de proponer que el desarrollo, como un estado deseable de una sociedad, es producto de una relación estrecha y dinámica de corresponsabilidad entre actores estratégicos para el territorio, dentro de los que se destaca la Universidad, por su posibilidad de interactuar en diferentes niveles con la sociedad a la que pertenece.

El presente documento consta de cuatro apartados incluyendo esta introducción: en la segunda parte se hace una contextualización de los 32 departamentos de Colombia y Bogotá D.C, a partir de la observación de las variables mayormente asociadas a tres enfoques del desarrollo: neoinstitucionalismo, capital humano y capital social; en el tercer apartado se describe el estado y el quehacer socialmente responsable de las Universidades, y finalmente se presenta a manera de conclusión, una discusión que vincula las capacidades de las universidades con los avances del territorio.

\section{MIRADAS REGIONALES DEL DESARROLLO EN COLOMBIA}

\subsection{Síntesis teóricas recientes del desarrollo}

El desarrollo se concibe como una situación socialmente deseable para las generaciones actuales y futuras, que se construye a partir del relacionamiento estratégico de diferentes actores, cuyas acciones y toma de decisiones están guiadas y estructuradas en aras de la transformación positiva de un territorio.

En consonancia, las propuestas teóricas del desarrollo cobran interés en la medida que identifican factores condiciones del bienestar, son generadoras de nuevas formas e interpretaciones de relaciones sociales, y permiten encaminar esfuerzos conducentes a progresos en las diferentes dimensiones del ser. En la Tabla 1 se expone una breve síntesis de tres enfoques teóricos propios del campo socioeconómico, que podrían considerarse explicativos modernos del desarrollo, y que dan cabida a repensar el rol de la Universidad como condicionante de las estructuras sociales y económicas. 
Tabla 1. Síntessis teóricas del desarrollo y su relación con la Universidad.

\begin{tabular}{|c|c|c|}
\hline $\begin{array}{l}\text { Enfoques teóricos } \\
\text { del Desarrollo }\end{array}$ & Postulados & Concepción de la Universidad \\
\hline $\begin{array}{l}\text { Nuevo Institucionalismo } \\
\text { (sociológico, económico) }\end{array}$ & $\begin{array}{c}\text { Reivindica la importancia de las } \\
\text { formas organizativas de la sociedad. } \\
\text { Apunta a identificar la estructura, el } \\
\text { funcionamiento y el rol de las } \\
\text { instituciones. } \\
\text { Se centra en el análisis de las normas } \\
\text { y acuerdos formales y convenciones } \\
\text { sociales que afectan el modo en que } \\
\text { los agentes toman decisiones. }\end{array}$ & $\begin{array}{l}\text { Marco de referencia cultural. } \\
\text { Se ajusta gradualmente en respuesta } \\
\text { a intereses personales, en función de } \\
\text { maximizar beneficios y reducir } \\
\text { costos de transacción. } \\
\text { Se rige por principios de } \\
\text { calidad y eficiencia. }\end{array}$ \\
\hline Capital Humano & $\begin{array}{l}\text { Se define como el conjunto de } \\
\text { conocimientos, habilidades y } \\
\text { destrezas que mejoran las } \\
\text { capacidades productivas de las } \\
\text { personas. } \\
\text { La educación formal y la experiencia } \\
\text { laboral son las principales fuentes de } \\
\text { capital humano; y la salud es un condi- } \\
\text { cionante del mismo. } \\
\text { La educación es una inversión en el } \\
\text { ser humano, y los efectos de esta se } \\
\text { consideran una forma de capital. }\end{array}$ & $\begin{array}{l}\text { Organización que genera, incorpora y } \\
\text { difunde avances del conocimiento en } \\
\text { los individuos. } \\
\text { Tiene un rol preponderante en el bien- } \\
\text { estar de los individuos, la } \\
\text { productividad de las empresas, y la } \\
\text { competitividad del territorio. } \\
\text { La educación superior es una } \\
\text { actividad especializada y costosa. }\end{array}$ \\
\hline Capital Social & $\begin{array}{l}\text { Consiste en un conjunto de valores, } \\
\text { actitudes y recursos propios de las } \\
\text { conexiones sociales, que sirve a los } \\
\text { individuos para alcanzar las metas de } \\
\text { sus acciones, y determinan la identi- } \\
\text { dad en/de un grupo social y su vínculo } \\
\text { con otros. }\end{array}$ & $\begin{array}{l}\text { Cumple un papel natural en la } \\
\text { generación, reproducción y } \\
\text { diversificación del capital social. } \\
\text { El capital social es insumo en los } \\
\text { procesos de la universidad. }\end{array}$ \\
\hline
\end{tabular}

Fuente: elaboración propia con base en Córdoba, (2017), García-Valdecasas, (2011), Villalobos \& Pedroza (2009), Chapela \& Jarillo, (2004), Rivas (2003).

El neoinstitucionalismo es un campo analítico que se centra en el estudio de las instituciones como unidad básica de la estructura social, y de su incidencia en el accionar y la toma de decisiones de los individuos. Desde este enfoque que cabe preguntarse sobre la estructura, el funcionamiento y el rol que asumen las instituciones como promotoras del bienestar individual y colectivo, y como dinamizadoras del desarrollo (Lipnicka \& Verhoeven, 2014), por lo que esta teoría ofrece un importante referente de análisis sobre el devenir de la Universidad (Cai \& Mehari, 2015): por una parte, constituyen un escenario de referencia cultural, en la medida que expresan un sentido de integralidad y significados subyacentes acerca del rol y la identidad del individuo en sociedad; pero también, actúan como instituciones formales, es decir, como organizaciones que son susceptibles a las tensiones internas y externas que se generan en el territorio, por lo que exhiben una naturaleza adaptativa y deben responder a las demandas individuales/sociales de calidad y eficiencia para sobrevivir.

La teoría del capital humano relieva las competencias económicas adquiridas por el individuo, en la medida que considera que el acervo de conocimientos especializados obtenidos por una persona, le permite mejorar su calidad de vida al posibilitarle el acceso a actividades laborales mejor remune- 
radas, así mismo, las empresas se ven beneficiadas de la mayor productividad laboral del individuo contratado y aumentan sus beneficios y, como resultado la sociedad se beneficia con un mayor cubrimiento de las necesidades de su población al existir más recursos para ello vía aumento de la producción y empleo. El rol de la Universidad bajo la teoría del capital humano es determinante: son los principales centros de generación y difusión del conocimiento, por lo que la educación se considera una inversión en capital humano (Schiller, 2008).

El capital social como teoría destaca la importancia de las redes sociales como potencializadoras de logros individuales (Daza, 2016) y promotoras de progreso social, toda vez que aspectos sociales como las normas, la confianza, la reciprocidad, entre otros, según Putnam (2000), facultan a las personas a aturar conjuntamente en procura de un bienestar común. Desde este enfoque teórico resalta la potencial capacidad de la universidad para construir y constituirse como capital social, por encima de cualquiera de las otras redes (Chapela \& Jarillo, 2004).

\subsubsection{Caracterización de la educación superior profesión departamental en Colombia}

Colombia es un país que está distribuido geopolíticamente en 32 regiones denominadas departamentos, más la capital del país, Bogotá. Socioeconómicamente, los polos de desarrollo se sitúan en Bogotá, Antioquia y Valle del Cauca, que actualmente concentran el $50 \%$ de la producción nacional, y el $40 \%$ de la población total del país. Esta situación es congruente con el panorama de educación superior que se presenta en la Tabla 2, en la que se observa que en estas mismas regiones hacen presencia el $60 \%$ del total de las Instituciones de Educación Superior (IES) del país, lo que refuerza la hipótesis planeada teóricamente en este estudio, sobre la importancia del rol de las Universidades en la promoción del desarrollo de las regiones. Cabe resaltar que, de las 301 IES en Colombia, tan solo la tercera parte ostentan el carácter de Universidad (104), aunque la matrícula en educación superior está representada proporcionalmente entre formación técnica profesional y tecnológica (51\%), y profesional (49\%). 
Tabla 2. Colombia. Población en edad de estudios superiores, número de IES y universidades y matrícula total y universitaria por sector, según departamento y distrito capital. 2017.

\begin{tabular}{|c|c|c|c|c|c|c|c|}
\hline \multirow{2}{*}{ Departamento } & \multirow{2}{*}{$\begin{array}{c}\text { Población } \\
\text { (17-24 años) }\end{array}$} & \multirow{2}{*}{ Total IES } & \multicolumn{2}{|c|}{ Universidades } & \multirow{2}{*}{$\begin{array}{c}\text { Total } \\
\text { matrícula }\end{array}$} & \multicolumn{2}{|c|}{ Matrícula } \\
\hline & & & Oficial & Privada & & Oficial & Privada \\
\hline Amazonas & 13.097 & 1 & 1 & & & & \\
\hline Antioquia & 898.613 & 42 & 2 & 10 & 455.970 & 92.139 & 150.994 \\
\hline Arauca & 41.025 & & & & & & \\
\hline Atlántico & 343.728 & 17 & 1 & 6 & 205.167 & 43.373 & 110.022 \\
\hline Bogotá D.C. & 1.057 .151 & 107 & 6 & 24 & 2.299 .987 & 257.658 & 495.845 \\
\hline Bolívar & 317.083 & 13 & 2 & 4 & 109.607 & 31.609 & 20.797 \\
\hline Boyacá & 168.811 & 4 & 1 & 2 & 76.409 & 45.606 & 18.019 \\
\hline Caldas & 127.088 & 6 & 2 & 3 & 63.708 & 31.771 & 21.170 \\
\hline Caquetá & 75.737 & 1 & 1 & & 17.503 & 17.285 & \\
\hline Casanare & 56.988 & 1 & & & 3.617 & & \\
\hline Cauca & 210.811 & 5 & 1 & & 57.904 & 30.770 & \\
\hline Cesar & 160.607 & 1 & 1 & & 31.478 & 31.338 & \\
\hline Chocó & 83.619 & 2 & 1 & & 23.128 & 19.072 & \\
\hline Córdoba & 263.649 & 3 & 1 & 2 & 51.039 & 31.041 & 19.235 \\
\hline Cundinamarca & 387.400 & 6 & 1 & 2 & 59.843 & 26.288 & 21.230 \\
\hline Guainía & 7.722 & & & & & & \\
\hline Guaviare & 18.397 & & & & & & \\
\hline Huila & 178.198 & 4 & 1 & & 36.003 & 22.527 & \\
\hline La Guajira & 146.615 & 2 & 1 & & 29.329 & 26.939 & \\
\hline Magdalena & 194.266 & 4 & 1 & 2 & 51.014 & 34.840 & 11.932 \\
\hline Meta & 140.353 & 2 & 1 & & 17.664 & 10.984 & \\
\hline Nariño & 254.807 & 5 & 1 & 1 & 60.175 & 23.185 & 12.595 \\
\hline \multicolumn{8}{|l|}{ Norte de } \\
\hline Santander & 207.656 & 5 & 2 & 1 & 105.368 & 91.070 & 3.878 \\
\hline Putumayo & 57.749 & 1 & & & 2.864 & & \\
\hline Quindío & 76.348 & 4 & 1 & 1 & 40.463 & 28.111 & 4.988 \\
\hline Risaralda & 128.210 & 7 & 1 & 2 & 59.471 & 27.203 & 12.984 \\
\hline \multicolumn{8}{|l|}{ San Andrés y } \\
\hline Providencia & 10.549 & 2 & & & 110 & & \\
\hline Santander & 284.215 & 16 & 1 & & 178.993 & 34.182 & 80.791 \\
\hline Sucre & 131.741 & 4 & 1 & & 37.363 & 9.452 & \\
\hline Tolima & 198.466 & 6 & 1 & 1 & 57.223 & 30.841 & 12.916 \\
\hline Valle del Cauca & 632.523 & 30 & 3 & 7 & 231.627 & 52.422 & 100.550 \\
\hline Vaupés & 7.210 & & & & & & \\
\hline Vichada & 12.280 & & & & & & \\
\hline Total & 6.892 .712 & 301 & 36 & 68 & 4.363 .027 & 1.049 .306 & 1.097 .946 \\
\hline
\end{tabular}

Fuente: Series de población - DANE, SNIES - Ministerio de Educación Nacional. 


\section{APROXIMACIONES A LA RESPONSABILIDAD SOCIAL UNIVERSITARIA (RSU) EN COLOMBIA}

Según Giraldo (2009), la responsabilidad social es un deber de todos los agentes y las organizaciones desde el rol que ocupan en la sociedad, en pro de dar respuesta a las situaciones problema del territorio. Desde esta perspectiva, las universidades se encontrarían en la obligación de contribuir, proteger, promover y consolidar los valores de la sociedad, en la búsqueda permanente por preservar y mejorar sus condiciones de vida y las de su entorno. Específicamente la Asociación Colombiana de Universidades (ASCUN) considera que:

Cuando se habla de RSU no sólo se hace referencia a la formación profesional de quienes pueden y van a ejercer un liderazgo en el manejo de las naciones y sus instituciones sociales, sino, además, a la creación de una sensibilidad especial y de una orientación para que dichos profesionales actúen con una ética preferencial por lo público y para que se desempeñen con una orientación hacia el bien común, y no sólo a favor del beneficio particular. (2011, p.13).

Cuando se hace una revisión sucinta de la compresión y ejercicio de la responsabilidad social de las universidades en Colombia, se encuentra que algunas ya han incursionado en la identificación, conceptualización y análisis de lo que representaría para su institución ser socialmente responsable y otras más, han diseñado y puesto en marcha modelos de responsabilidad social para sus universidades. Al respecto Cortés (2013) señala que, si bien la RSU no es un concepto reciente en Colombia, si se visualiza como una corriente innovadora, lo que ratifica su vigencia y necesidad de profundizar en su investigación.

No obstante lo anterior, aún en Colombia prevalece como común denominador las acciones de extensión por sobre las socialmente responsables, esto se puede evidenciar en la Tabla No.3, en las que se presenta una selección de universidades públicas para las tres principales regiones del país, y se observa que si bien todas cuentan con un área de extensión, no es así sobre áreas de RSU, aún en el caso de la Universidad de Antioquia las actividades de RSU están enmarcadas en el ámbito de la extensión, y si bien en la Universidad del Valle las actividades de extensión se encuentran enmarcadas en la RSU, no hay evidencia explicitica de la existencia de la existencia de políticas, referentes o normativas que la definan o guíen.

Tabla 3. Colombia. Selección de universidades públicas según región. 2018.

\begin{tabular}{|c|c|c|c|}
\hline Región & Universidad & Área de extensión & Área de RSU \\
\hline Bogotá D.C. & Universidad Nacional & Si & No \\
\hline Antioquia & Universidad de Antioquia & Si & Si \\
\hline Valle del Cauca & Universidad del Valle & Si & No \\
\hline
\end{tabular}

Fuente: elaboración propia.

\section{DISCUSIÓN Y REFLEXIONES FINALES}

Como se pudo inferir a lo largo de este escrito, ciertamente en Colombia como en muchas otras partes del globo, los beneficios del dinamismo económico no permean por igual ni suficientemente todas las regiones del territorio, de ahí la importancia, primeramente, de promover la diversificación espacial de aquellos elementos que se consideran potenciadores del desarrollo, que tal como ilustran los nuevos enfoques teóricos del desarrollo, estarían en buena medida asociados al rol que desempeñan las Universidades. 
Si bien las universidades en Colombia son un cuerpo representativo de las IES del país, tanto en número como en matrícula, aún existen departamentos en el país que adolecen de representación universitaria, siendo estos los más rezagados económicamente. Es necesario que en estos departamentos exista presencia de universidades públicas como ejes dinamizadores del desarrollo, por lo que una solución es llevarlas bajo un modelo de ampliación de cobertura, en alianza con instituciones ya creadas, o en la modalidad semipresencial o virtual.

Bajo los tres enfoques teóricos, las universidades son una de las instituciones más importantes en el marco del desarrollo, como promotor de capital social, como generador de capital humano y como base cultural de una sociedad. Estos postulados están claramente identificados en las concepciones que sobre RSU se están gestando en el país, pero que aún no han logrado concretarse en una construcción colectiva de política nacional o políticas regionales, que les permitan a las universidades hablar un mismo lenguaje y propender por un bienestar común.

La RSU en Colombia como modelo de gestión universitaria aún es incipiente. Son pocas las universidades que realmente han implementado desde la visión estratégica de su institución, políticas regentes para la orientación de un accionar socialmente responsable, y aquellas que han avanzado en el ejercicio de la RSU, aún es abordado desde otras áreas de gestión, o se limita a las actividades de extensión. Por lo pronto, se encuentra alicientes en el creciente número de investigaciones sobre el tema que se vienen desarrollando desde las universidades, con lo que se esperaría que en los próximos años, se empiece a visibilizar un accionar responsable de las universidades, que no sólo permee sino que oriente todas las funciones sustantivas de la Universidad.

\section{REFERENCIAS BIBLIOGRÁFICAS}

Asociación Colombiana de Universidades. (2011). Responsabilidad Social Universitaria. Pensamiento Universitario, 21.

Cai, Y. y Mehari, Y. (2015). The Use of institutional theory in higher education research. In Theory and Method in Higher Education Research III, Jeroen Huisman and Malcolm Tight (Eds), pp.1-25. Bingley: Emerald.

Chapela, M. y Jarillo, E. (2004). El capital social en el futuro de la sociedad. Reencuentro, (40), pp.1-13.

Córdoba, M. (2017). El neoinstitucionalismo como paradigma científico. Revista Latinoamericana de Políticas y Acción Pública, 4(1), pp.9 - 46.

Departamento Administrativo Nacional de Estadísticas. (2017). Colombia. Estimaciones 1985- 2005 y Proyecciones 20052020 nacional y departamental desagregadas por sexo, área y grupos quinquenales de edad. Disponible en: http:// www.dane.gov.co/index.php/estadisticas-por- tema/demografia-y-poblacion/series-de-poblacion.

Daza, L. (2016). The role of social capital in students' perceptions of progress in higher education. Educational Research and Evaluation, 22 (1-2), pp.65-85.

García-Valdecasas, J. (2011). Una definición estructural de capital social. Revista Hispana para el Análisis de Redes Sociales, 20(6), pp.132 - 160.

Giraldo, S. (Comp.) (2009). Responsabilidad Social Universitaria. Estudio de caso sobre Paz y Competitividad de la Universidad Autónoma de Manizales. Manizales:

Lipnicka, M. y Verhoeven, J. (2014). The application of new institutionalism and the resource dependence theory for studying changes in universities within Europe. Roczniki Nauk Społecznych, 6/42 (4), pp.7-30.

López, V. (2017). Modelo de responsabilidad social universitaria para la Facultad de Administración de la Universidad Nacional de Colombia Sede Manizales. Tesis de Maestría.

Ministerio de Educación Nacional. (2018). Matriculados en educación superior. Disponible en: https://www.mineducacion. gov.co/sistemasdeinformacion/1735/w3-article-212400.html. 
Putnam, R. (2000). Bowling alone: the collapse of and revival of American community. Nueva York: Simon and Shuster.

Rivas, J. (2003). El neoinstitucionalismo y la revalorización de las instituciones. Reflexión Política, 5(9), pp. 37 - 46.

Schiller, T. (2008). Human capital and higher education: How does our region fare?. Business Review, Q 1, pp. 16 - 26.

Villalobos, G., y Pedroza, N. (2009). Perspectiva de la teoría del capital humano acerca de la relación entre educación y desarrollo económico. Tiempo de Educar, 15 (29), pp. 273 - 306. 